\title{
Les Cours de Comptes et la tutelle environnementale
}

\section{Os Tribunais de Contas e a tutela ambiental}

\section{The Courts of Accounts and the environmental protection}

\author{
Grace Ladeira Garbaccio* \\ Marie Tanchon \\ Renato Luís Bordin de Azeredo"*t* \\ Jeferson Dytz Marin ${ }^{*+*+*}$
}

\section{Resumé}

Les Cours de Comptes du Brésil ont une nature juridique sui generis. Ce sont des organes autonomes et d'une haute importance dans le contexte juridique constitutionnel brésilien. Ils excercent la fonction d'"auxiliaire" au Pouvoir Législatif dans le contrôle des actes de la gestion publique. Ils ont une large compétence d'analyse dans le cadre de l'environnement, et peuvent vérifier des organes soumis à son contrôle leurs actions sous l'aspect de l'économie et de légitimité. Ces tribunaux possèdent en plus de cela des instruments des modes d'action préventives calqués sur les principes de prévention et de précaution qui justifient l'emploi de la tutelle inhibitoire, avec le but d'éviter l'occurrence de dégâts à l'environnement. C'est par le biais de prises de comptes spéciaux, de la non-exécution d'actes normatifs inconstitutionnels, du pouvoir général de protection et du terme d'adoption de providences que doivent agir de forme à inhiber tout outrage à l'environnement. À travers une synthèse scientifique, cet article utilise la méthode de la recherche déductive et exploratoire, avec une contribution à l'étude prospectif du droit positif, basée sur l'analyse des références d'articles et des études bibliographiques.

Mots-clés: Politiques publiques. Environnement. Tutelle inhibiteur de l'environnement. Cour des Comptes.

\section{Resumo}

Os Tribunais de Contas no Brasil possuem uma natureza juridica sui generis. São órgãos autônomos e sobranceiros no contexto jurídico constitucional brasileiro. Exercem função de "auxílio" ao Poder Legislativo no controle dos atos da gestão pública. Tem ampla competência de análise em matéria ambiental, podendo aferir dos órgãos submetidos ao seu controle as suas ações sob o aspecto da economicidade e legitimidade. Possuem ainda instrumentos de atuação preventiva calcados nos princípios da prevenção e precaução que justificam o emprego da tutela inibitória, com o escopo de evitar a ocorrência de danos ao meio ambiente. Através de tomadas de contas especiais, da negativa de executoriedade de atos normativos inconstitucionais, do poder geral de cautela e do termo de adoção de providências é que devem atuar de forma a inibir qualquer ultraje ao meio ambiente. Por meio de uma síntese científica, este artigo utiliza o método de pesquisa dedutiva e exploratória, com uma contribuição para o estudo prospectivo do direito positivo, com base na análise de referências de artigos e estudos bibliográficos.

Palavras-chave: Politicas Públicas. Meio Ambiente. Tutela inibitória ambiental. Tribunal de Contas.

\section{Abstract}

The Brazilian Courts of Accounts have a sui generis legal nature. They are autonomous and outstanding bodies in the Brazilian constitutional legal context. They have the function of "assisting" the Legislative Power in controlling public management

Doutora e mestre em Direito pela Universidade de Limoges e título reconhecido pela Universidade Federal de Santa Catarina (UFSC). Professora do Programa Stricto Sensu do Mestrado Acadêmico em Direito Constitucional e do Mestrado profissional em Administração Pública do Instituto Brasileiro de Ensino, Desenvolvimento e Pesquisa (IDP). Professora do programa lato sensu da Fundação Instituto de Administração (FIA), ESPM, Escola de Direito do Brasil (EDB), Instituto Brasileiro de Ensino, Desenvolvimento e Pesquisa (IDP) em Brasília - DF - Brasil. E-mail : glgarbaccio@ hotmail.com.

* Coordinatrice et Professionnelle de recherche à la Chaire de recherche et d'innovation Goldcorp en droit des ressources naturelles et de l'énergie. Université Laval. Québec - Canadá. Email: marie.tanchon.1@ulaval.ca.

... Mestre em Direito Ambiental pela Universidade de Caxias do Sul (UCS). Especialista em Processo Civil e Direito Civil pelo Instituto de Desenvolvimento Cultural e Especialista em Direito Público pela Pontifícia Universidade Católica do Rio Grande do Sul (PUC-RS). Auditor Público Externo do Tribunal de Contas do Estado do Rio Grande do Sul. Caxias do Sul - RS - Brasil. E-mail: renazeredo@hotmail.com.

.... Doutor em Direito pela Universidade do Vale do Rio dos Sinos (UNISINOS-RS). Advogado. Coordenador e professor do Programa de Mestrado e Doutorado em Direito da Universidade de Caxias do Sul (UCS-RS). Caxias do Sul - RS - Brasil. E-mail: jdmarin@ucs.br. 
acts. It has broad competence of analysis in environmental matters, being able to verify from the organs submitted to its control their actions in the aspect of economy and legitimacy. They also have preventive action instruments based on the principles of prevention and precaution that justify the use of inhibitory protection, with the scope of preventing the occurrence of damage to the environment. It is through special accountability, the denial of enforceability of unconstitutional normative acts, the general power of caution and the term of action that they must act to inhibit any outrage on the environment. Through a scientific synthesis, this article uses the method of deductive and exploratory research, with a contribution to the prospective study of positive law, based on the analysis of references of articles and bibliographic studies.

Keywords: Public Policies. Environment. Inhibitory environmental protection. Court of Accounts.

\section{Introduction}

Une protection inhibitrice de l'environnement suppose une action rapide des agences d'État chargées de la surveillance et du contrôle des entités fédératives dans la formulation et l'exécution de leurs tâches constitutionnelles. Il est donc nécessaire que les cours de comptes établissent, dans les règles qui organisent leurs activités, des mécanismes permettant une action préventive.

Dans le domaine judiciaire, le sujet est bien développé dans la doctrine, en particulier dans le cas de la loi sur l'action publique civile, qui est l'un des principaux instruments de protection de l'environnement actuellement disponibles. On ne peut plus en dire autant des cours de comptes, dans lesquelles l'attention n'est toujours pas portée à la question. Toutefois, ces organes disposent de plusieurs mécanismes, reconnus dans la doctrine et la jurisprudence, en particulier de la Cour suprême fédérale, ainsi que dans ses lois organiques et son règlement intérieur qui permettent cette action préventive dans le domaine de la protection de l'environnement.

Une analyse des instruments suivants est actuellement en cours: exercice du pouvoir général de prudence, négation du caractère exécutoire de la loi ou de l'acte normatif, adoption de mesures et prise de compte particulière. Cependant, nous discuterons d'abord de la manière dont les cours de comptes agissent et de l'émergence d'une action préventive.

En ce sens, on peut clairement affirmer que les cours de comptes disposent d'une légitimité et d'instruments juridiques pour la mise en œuvre d'actions préventives en faveur de la protection de l'environnement, qui ne se limitent pas à la contrainte, en punissant les actes déjà accomplis.

Ainsi une réflexion sur le Cours de Comptes du Brésil et la protection de l'environnement s'impose. Cet article est basé sur une recherche exploratoire-descriptive et bibliographique qui combine l'état des lieux juridiques dans le cadre du droit de l'environnement.

\section{La manière d'agire de la Cour de Comptes}

Les Cours de Comptes, dans l'exercice de leurs compétences, peuvent procéder à un examen officiel du dossier qui leur est attribué, par le biais d'audits ou d'inspections, ${ }^{1}$ ou même agir par provocation des plus légitimes.

\footnotetext{
“Article 71. Le contrôle externe, sous la responsabilité du Congrès national, sera exercé avec l'assistance de la Cour fédérale des comptes, qui est chargée: IV - d'exercer, de sa propre initiative, la Chambre des députés, le Sénat fédéral, la Commission technique ou enquête, inspections et audits de nature comptable, financière, budgétaire, opérationnelle et patrimoniale, dans les unités administratives des pouvoirs législatif, exécutif et judiciaire, et dans les autres entités visées au point II;"

CF/88 - "Art. 74. Les pouvoirs législatif, exécutif et judiciaire maintiendront, de manière intégrée, un système de contrôle interne visant à:

I - évaluer la réalisation des objectifs prévus dans le plan pluriannuel, l'exécution des programmes gouvernementaux et les budgets de l'Union;

II - prouver la légalité et évaluer les résultats, quant à l'efficacité et l'efficience, de la gestion budgétaire, financière et patrimoniale dans les organes et entités de l'administration fédérale, ainsi que l'utilisation des ressources publiques par des entités de droit privé:

III - exercer un contrôle sur les opérations de crédit, les cautions et garanties, ainsi que sur les droits et les actifs de l'Union;

IV - soutenir le contrôle externe dans l'exercice de sa mission institutionnelle.

$\S 1$ - Les responsables du contrôle interne, dès la connaissance de toute irrégularité ou illégalité, en informeront la Cour fédérale des comptes, sous peine de responsabilité solidaire.

$\S 2$ - Tout citoyen, parti politique, association ou syndicat est un parti légitime pour, selon la loi, dénoncer des irrégularités ou des illégalités devant la Cour fédéral des comptes. 75 du CF/88 s'applique aux autres TC].

"Loi 8 666/1993: Art. 113. Le contrôle des dépenses résultant des contrats et autres instruments régis par la présente loi sera effectué par la Cour de comptes compétente, conformément à la législation pertinente, avec les organes intéressés de l'administration chargés de démontrer la légalité et la régularité des dépenses et de l'exécution, conformément aux dispositions de la Constitution et sans préjudice du système de contrôle interne qui y est prévu.

Paragraphe 1. Tout soumissionnaire, contractant ou personne physique ou morale peut représenter la Cour des comptes ou les organismes faisant partie du système de contrôle interne contre les irrégularités dans l'application de la présente loi, aux fins des dispositions du présent article."
} 
L'une des formes d'action consiste à examiner les faits issus de la représentation. Et il existe plusieurs dispositions du système juridique permettant de participer à la formation d'un processus de vérification de la régularité des actes administratifs. ${ }^{2}$

En ce sens, la possibilité d'initier les diverses formes d'action de ces organes est large, à travers des audits, des inspections et les instruments fournis dans le cadre de l'activité courante des tribunaux.

\subsection{La justification des mesures de tutelle inhibiteur dans les organes de surveillance et de contrôle}

Le besoin d'une action préventive dans la protection des ressources publiques, en inhibant et en corrigeant les écarts avant que la corruption ne s'épuise, est un impératif de sécurité pour la mise en œuvre des droits fondamentaux. En ce sens, comme le fait remarquer à juste titre Rizzo Junior, en termes de rachat de droits fondamentaux violés, en raison du manque absolu de ressources financières, rôle de la purification symbolique (RIZZO JÚNIOR, 2009, p. 14).

L'état de léthargie de la population, incapable d'exiger une attitude différente de celle présentée sur la scène nationale, est paradoxal. Cela signifie que peu se soucient de ce qui traduit le nerf de tout, la politique. Plutôt que d'orienter leurs actions vers la cessation des niveaux exacerbés de corruption, ils se tournent de plus en plus vers des délits susceptibles d'empêcher l'accès aux îles de la prospérité.

Ce que la population brésilienne veut et attend, c'est une véritable transformation des structures sociales du pouvoir, qui permette d'élaborer des politiques publiques solides et durables, de manière à permettre le développement d'une société dans laquelle les services publics sont de qualité et non pas simplement fictifs, au point d'essayer d'exercer des ordres constitutionnels que formellement ou à temps, comme dans l'éducation, la santé, la sécurité et l'environnement.

Une lumière au bout de ce tunnel a été vue le 7 septembre 2011, lorsqu'elle a été rapportée dans les médias, lors de manifestations de masse contre la corruption. Date symbolique pour le pays dans lequel a été célébrée une autre année de son indépendance. II semble que le peuple reprenne la participation nécessaire au sauvetage de la citoyenneté participative et prenne une position sérieuse et claire contre les maux causés par la corruption et revendique une position plus conforme aux principes de la République.

Le passage suivant du travail de Rizzo Junior $(2009$, p. 13) reproduit bien la situation:

[...] Nous avons la même vision terrifiante des catastrophes qui s'empilent à nos pieds, le même excès de lucidité de nos propres maladies et le même sentiment d'impuissance. La catastrophe sans fin que nous envisageons dans sa véritable dimension n'est perçue que comme un fait isolé justifiant des sanctions tardives et inutiles. La constatation d'actes répréhensibles et la punition possible du coupable nous donne la fausse impression que la punition certaine et inexorable suffit à compenser les pertes subies par l'État. Toutefois, les réparations que l'exécution de la justice peut apporter ne sont pas plus grandes que le réconfort de la mère qui assiste à la condamnation du meurtrier de son fils. La condamnation du coupable ne lui rendra pas l'enfant et ne soulagera pas sa douleur, car les moyens disponibles dans le système normatif n'apporteront pas à la société la réalisation de ses attentes justes, frustrée par la corruption ou l'incompétence de ses représentants.

2 CF / 88 - "Art. 74. Les pouvoirs législatif, exécutif et judiciaire maintiendront, de manière intégrée, un système de contrôle interne visant à: I - évaluer la réalisation des objectifs prévus dans le plan pluriannuel, l'exécution des programmes gouvernementaux et les budgets de l'Union; II - prouver la légalité et évaluer les résultats, quant à l'efficacité et l'efficience, de la gestion budgétaire, financière et patrimoniale dans les organes et entités de l'administration fédérale, ainsi que l'utilisation des ressources publiques par des entités de droit privé;

III - exercer un contrôle sur les opérations de crédit, les cautions et garanties, ainsi que sur les droits et les actifs de l'Union;

IV - soutenir le contrôle externe dans l'exercice de sa mission institutionnelle.

Paragraphe 1 - Les responsables du contrôle interne, dès la connaissance de toute irrégularité ou illégalité, en informeront la Cour fédérale de comptes, sous peine de responsabilité solidaire.

$\S 2$ - Tout citoyen, parti politique, association ou syndicat est un parti légitime pour, selon la loi, dénoncer des irrégularités ou des illégalités devant la Cour fédéral des comptes. 75 du CF/88 s'applique aux autres TC].

"Loi 8 666/1993: Art. 113. Le contrôle des dépenses résultant des contrats et autres instruments régis par la présente loi sera effectué par la Cour de comptes compétente, conformément à la législation pertinente, avec les organes intéressés de l'administration chargés de démontrer la légalité et la régularité des dépenses et de l'exécution, conformément aux dispositions de la Constitution et sans préjudice du système de contrôle interne qui y est prévu.

Paragraphe 1. Tout soumissionnaire, contractant ou personne physique ou morale peut représenter la Cour de comptes ou les organismes faisant partie du système de contrôle interne contre les irrégularités dans l'application de la présente loi, aux fins des dispositions du présent article." 
La transformation doit surtout concerner l'action des institutions de l'État. On ne voit plus le pouvoir judiciaire épuiser les limites de ses possibilités dans une tentative futile de contrôler une gestion de plus en plus frauduleuse, "ni la Cour des comptes simplement en tant que comptable, dans sa fonction de démembrer déjà le corps sans la vie des aspirations et des besoins fondamentaux de l'être humain" (RIZZO JÚNIOR, 2009, p. 18).

Il est indéniable qu'un contrôle social réellement efficace et sécurisé ne peut être réalisé que par l'intermédiaire de la Cour des comptes. Il est une entité neutre et indépendante, naturellement conçue pour filtrer et évaluer les informations nécessaires à la stimulation de la société et donner un avis sur le sort des richesses qui lui sont demandées (RIZZO JUNIOR, 2009, p. 21).

Rosane Heineck Schmitt a adopté une approche intéressante en ce qui concerne l'étendue des pouvoirs implicites, selon laquelle toute attribution / compétence déléguée par la Constitution inclut le droit / pouvoir d'exercer tous les pouvoirs exprès et tous les autres moyens nécessaires autorisés par la Constitution pour l'exécution des pouvoirs qui lui sont conférés/délégués (SCHMITT, 2006).

Par conséquent, l'exercice effectif des pouvoirs constitutionnels conférés aux organes de surveillance et de contrôle, tels que les cours de comptes, en matière de défense et de protection de l'environnement, en utilisant les instruments accélérés déjà à leur disposition, reconnus même par la Cour Suprême Fédéral, comme dans le cas du pouvoir général de prudence, de la théorie des pouvoirs implicites, de la possibilité de refuser l'applicabilité de la loi ou de l'acte normatif inconstitutionnel, de la comptabilité spéciale, ainsi que de l'application de la durée d'adoption de mesures, fondée sur: Les articles 71, IX et X de la Constitution Fédérale, l'exigence de critères de durabilité dans les offres, la demande de dossier d'appel d'offres et la possibilité de déterminer les modifications à apporter au respect effectif de la loi - y compris en exigeant que l'Étude d'Impact Environnementale (EIE) et le Rapport d'Impact de l'Environnement (RIMA) soient absents ou non complément, ce qui est insuffisant, comme l'indique le paragraphe 2 de l'article $113^{3}$ de conduisent nécessairement à ce changement culturel proposé et assurent une protection de l'environnement plus conforme aux attentes de ces institutions.

Et la performance dans le domaine de l'environnement fait de plus en plus l'objet de préoccupations par les Cours de Comptes, car l'environnement est un bien public de la communauté et est constamment menacé de dégradation, ce qui nuit à la qualité de la vie.

En fait, selon Andreza Cristina Stonoga (2007, p. 34), "la préoccupation mondiale vis-à-vis de l'environnement découle d'un facteur prépondérant, à savoir "la protection de la vie humaine".

Rétablir l'environnement dans lequel il était après avoir été victime d'une agression est difficile, voire impossible. Pour cette raison, la protection inhibitrice devrait être privilégiée par le biais d'organismes judiciaires et administratifs de protection de l'environnement.

Le traitement des faits dans la société actuelle est tel que l'attente des protections traditionnelles et qui, dans la plupart des cas, ont été qualifiées de réparatrices ne constituent plus la solution adéquate et nécessaire pour faire face au problème.

Ainsi, il faut souligner la nécessité d'une analyse des politiques publiques à la lumière des aléas actuels, tels que l'innovation technique et l'évolution scientifique, susceptibles de réorganiser toute une direction de l'action publique. Être employé doit toujours aboutir aux résultats à atteindre. En ce sens, s'il est possible d'atteindre l'objectif proposé par des moyens plus efficaces et moins pénibles, il faut rediriger les actions.

\footnotetext{
“Article 113. Le contrôle des dépenses résultant des contrats et autres instruments régis par la présente loi sera effectué par la Cour de comptes compétente, conformément à la législation pertinente, avec les organes intéressés de l'Administration chargés de démontrer la légalité et la régularité des dépenses et de l'exécution, aux termes de la Constitution et sans préjudice du système de contrôle interne qui y est prévu.

$\S 1$ Tout soumissionnaire, contractant ou personne physique ou morale peut représenter la Cour de comptes ou les organes faisant partie du système de contrôle interne contre les irrégularités dans l'application de la présente loi, aux fins des dispositions du présent article.

Paragraphe 2. Les Cours de Comptes et les organes faisant partie du système de contrôle interne peuvent demander l'examen, jusqu'au jour ouvrable précédant immédiatement la date de réception des propositions, une copie de l'avis d'offre déjà publié, avec l'obligation des organes ou entités de direction intéressés par l'adoption de mesures correctives pertinentes qui, à la suite de cet examen, sont déterminées. (Libellé donné par la loi ${ }^{\circ} 8.883$, de 1994)."
} 
Dans la perspective de la mise en place d'une règle de droit socio-environnementale, Sarlet et Fensteisefer désignent comme un instrument de sa mise en œuvre l'étude préalable d'impact sur l'environnement (art. 225, $\S 1^{\circ}$, IV, de la Constitution Fédérale) requise pour l'installation du travail ou de l'activité en cause ou potentiellement dangereux causer une dégradation importante de l'environnement. Pour les auteurs, il s'agit d'un mécanisme d'ajustement de l'activité économique, ainsi que d'un devoir fondamental qui limite les droits de propriété et la libre entreprise des acteurs économiques privés, basés sur le développement durable (SARLET; FENSTERSEIFER, 2010, p. 22-23).

Cependant, comme cela a été bien souligné, la construction d'une action administrative doit peser les intérêts en jeu afin de parvenir à la mise en œuvre du principe de la dignité humaine. La rapidité des transformations sociales commence à exiger des institutions des actions susceptibles d'accompagner cette nouvelle forme de relation sociale.

Le droit à l'environnement ne trouve pas un seul bénéficiaire, de sorte que les actions individuelles ne se prêtent pas à la protection de l'environnement ou sont insuffisantes compte tenu de la diversité des situations à affronter. C'est dans ce sens que les institutions chargées de garantir l'état de droit qui permet une existence digne sont de la plus haute importance pour la protection des intérêts qui imprègnent la sphère individuelle. Pour cette raison, outre les organes exécutifs liés au pouvoir exécutif, les organes de contrôle et de surveillance peuvent agir de manière à minimiser autant que possible les effets, s'ils ne cessent pas de porter atteinte à l'environnement.

Dans le domaine judiciaire, il est fait référence à la loi sur l'action publique civile, qui constitue un jalon en matière de protection des intérêts homogènes diffus, collectifs et individuels. Cependant, ce qui a été observé ces dernières années, bien qu'aucune statistique ne permette une meilleure analyse à ce sujet, c'est le ministère public, le principal organe qui a engagé des poursuites dans ce domaine. Outre le ministère public, la légitimation du bureau du défenseur public représente une avancée majeure dans le développement d'une culture de mesures de protection visant à prévenir la dégradation par rapport à la tentative souvent infructueuse de récupération.

Outre les mesures prises dans le domaine judiciaire, il existe un champ d'action qui comprend une participation spéciale des Cours de Comptes en tant qu'organe de surveillance. L'analyse de la bonne structuration des organes exécutifs fait partie de ce champ de contrôle. Une analyse précise de la réactivité des organes chargés de mener des actions de protection et de défense de l'environnement est nécessaire. Ont-ils une structure personnelle et financière pour leur travail? Comment avez-vous répondu aux demandes sociales dans ce domaine? Enfin, ces organes sont responsables de la vérification et du contrôle des entités chargées de l'exécution directe de la protection de l'environnement.

Une autre action dans ce domaine concerne l'utilisation efficace des instruments juridiques mis à la disposition des entités fédératives. II s'agit d'accélérer les actions et de gérer les actions permettant une protection rapide. Dans ce domaine, l'action civile publique peut et doit être soumise à l'entrée d'autres entités légitimes face à la dégradation de l'environnement relevant de leurs compétences. Le rôle des organes de poursuite judiciaire et de contrôle interne revêt une importance fondamentale dans ce domaine.

Par conséquent, obtenir une injonction dans le cadre d'une action civile publique permet la cessation immédiate du dommage. Et l'atteinte de ce désir est une obligation de l'entité en charge de l'exécution des tâches publiques.

II n'existe pas seulement la possibilité mais aussi le devoir des Cours de Comptes d'inviter les administrateurs à provoquer les instances judiciaires pour l'ouverture d'actions civiles publiques dans le domaine de l'environnement, sans attendre l'action du ministère public, comme moyen de diffuser une culture respectueuse de l'environnement.

Les comités techniques sont compétents pour examiner l'économie et la légitimité des actions des agences soumises à leur contrôle. Dans ce domaine, souvent l'omission, l'absence d'action préventive engendre des coûts importants en ce qui concerne la nécessité d'une action réparatrice de la part des organismes publics chargés de l'application des lois. Pour cette raison, le comportement du gestionnaire 
dans le domaine de l'environnement peut et doit être mesuré afin qu'un jugement sur les comptes publics puisse être formé. S'il n'a pas pris les mesures appropriées pour protéger l'environnement, exhortant les instances juridiques soumises à sa hiérarchie à prendre les mesures de protection nécessaires pour prévenir la dégradation de l'environnement, leurs comptes peuvent et doivent être rejetés.

II convient de noter que cette action du responsable relève du domaine des comptes de gestion et est sujette à un arrêt définitif de la Cour des comptes, du moins dans le cadre de ses compétences, et ne peut être contrôlée que par le pouvoir judiciaire, mais de manière restreinte, comme indiqué la jurisprudence.

Par conséquent, comme cela a été amplement démontré, les comptes, lorsqu'ils sont jugés par les cours de comptes dans les limites de leurs compétences limitées, entraînent directement une série de conséquences pour le gestionnaire, telles que l'inéligibilité, ${ }^{4}$ la constitution de titres de dirigeants avec le montant des dommages causés à la Cours de Comptes, permettant à la valeur de retourner dans les coffres publics.

On ne peut perdre de vue la connectivité protectrice qui ne se limite pas aux générations actuelles mais aussi aux générations futures. En fait, il convient de noter que s'il n'y avait pas auparavant de connaissances suffisantes sur la possibilité d'une dégradation de l'environnement ou son étendue, elle pourrait menacer l'existence même des générations futures, sans parler de la possibilité de contact avec diverses espèces d'animaux ou avec des animaux. Les environnements naturels et les écosystèmes ayant cessé d'exister, il existe aujourd'hui un consensus scientifique sur les effets de l'action humaine sur l'environnement. Pour cette raison, il doit et doit adopter une position éthique à l'égard de la préservation des écosystèmes et des espèces, permettant ce contact futur et même une meilleure compréhension de leur importance existentielle.

\section{L'exercice du pouvoir général de prudence par les Cours de Comptes}

Le pouvoir général de prudence est l'institut qui permet de garantir l'efficacité des décisions rendues dans les procédures relevant de sa compétence, dans la mesure où il garantit une issue utile au jugement final.

Le pouvoir général de prudence vise à garantir la possibilité de sa réalisation future, en tenant compte d'un besoin de sécurité face à une situation pertinente, afin qu'elle ne périsse pas.

La mesure de précaution est de nature précaire et vise uniquement à assurer un résultat utile à la fin du processus, qu'il soit administratif ou judiciaire. Il se caractérise par son instrumentalité et sa recevabilité. II vise à garantir une situation exécutoire (MARINONI, 2008, p. 739).

II ne faut pas oublier que l'exercice du pouvoir général de prudence est soumis au principe de l'application régulière de la loi $^{5}$ dans la mesure où il vise le processus général, qu'il soit administratif ou judiciaire. L'octroi de l'injonction ne supprime pas le droit à une défense contradictoire et large, il ne diffère que par le moment où il s'exerce en raison de la nécessité des valeurs qui visent à assurer.

En outre, il convient de noter que l'octroi d'une injonction, conformément à la jurisprudence de la Cour suprême, n'est justifié que lorsque les exigences énoncées à l'article 7, II de la loi n 1.533/1951 sont configurées, à savoir: la plausibilité juridique de la créance et le risque de blessure irréparable ou difficile à réparer.

Les décisions rendues doivent être dûment motivées et démontrer les éléments qui les ont provoquées, sous peine de nullité.

\footnotetext{
$4 \quad$ "Loi complémentaire $n^{\circ}$ 64: Art. 1 Ne sont pas éligibles:

I - pour tout poste:

$[\ldots]$

g) ceux dont les comptes relatifs à l'exercice de fonctions ou fonctions publiques sont rejetés par une irrégularité insurmontable qui constitue un acte volontaire d'improbabilité administrative, et par une décision irrévocable de l'organe compétent, à moins qu'elle n'ait été suspendue ou annulée par le pouvoir judiciaire, pour les élections qui ont lieu dans les 8 (huit) années suivantes, à compter de la date de la décision, en application des dispositions du point II de l'art. 71 de la Constitution fédérale, à tous les ordonnateurs aux frais, sans exclure les représentants qui ont agi dans cette condition; (Libellé donné par la loi complémentaire n ¹35, 2010)."

5 Article $5^{\circ}$, LIV, de la CF/88.
} 
Pour l'exercice des pouvoirs constitutionnellement établis, les CT ont besoin de procédures procédurales pour garantir l'efficacité de leurs décisions, sous peine de ne pas atteindre l'objectif de leur existence, à savoir le contrôle des comptes publics. Si tel n'était pas le cas, les droits garantis par la Constitution s'épuiseraient.

La Cour Suprême Fédérale a déjà eu l'occasion de se prononcer sur la possibilité d'exercer le pouvoir général de prudence des cours de comptes, en rendant un arrêt en bref de Mandamus 24.510-7/DF, formé contre une décision de la Cour des comptes du Union qui avait décidé de suspendre les offres, compte tenu des forts indices d'irrégularités. Le rapporteur, dans son vote, a déclaré:

[...] le pouvoir de précaution constitue également le domaine des attributions institutionnelles de la Cour de Comptes puisqu'il a pour instruction instrumentale de rendre effective l'exercice par cette Haute Cour des pouvoirs multiples et pertinents que lui confère directement le même texte même de la Constitution de la République. Cela signifie que l'attribution de pouvoirs explicites à la Cour de Comptes au sens de l'art. 71 de la Loi fondamentale de la République, il est supposé que, même implicitement, la possession de moyens permettant l'adoption de mesures conservatoires destinées à donner une réelle efficacité à ses délibérations finales, permettant ainsi de neutraliser les situations de nocivité actuel ou imminent, au trésor public. [...] En effet, l'exercice du pouvoir de prudence de la Cour de Comptes a pour but de garantir l'utilité même de la décision finale à prendre par celle-ci afin d'éviter tout retard dans l'appréciation du fond de la question posée pour avoir affecté, compromis et frustré le résultat final de l'examen de la controverse.

De toute évidence, le même raisonnement devrait être appliqué lors de l'examen des questions environnementales, compte tenu des dommages subis par la communauté et le public en raison des compensations élevées à supporter, si elle ne dit rien sur les mesures de protection de l'environnement, ainsi que dommages directs au patrimoine environnemental. En outre, cela relève de la compétence de ces organes. A titre d'exemple, il est fait mention de la suspension d'une offre pour des travaux majeurs dans lesquels l'étude d'impact préalable sur l'environnement n'a pas été réalisée. En fait, cette affirmation est fondée sur les dispositions de l'article 225 , paragraphe $1^{\circ}$, IV, de la Constitution fédérale, qui prévoit l'étude de l'impact environnemental antérieur.

L'action de précaution des Cours de Comptes repose sur la théorie des pouvoirs implicites, selon Wedy:

De même, la Cour de Comptes, en appliquant la théorie des pouvoirs implicites à l'art. 71 de la Constitution Fédérale peuvent, par mesures conservatoires, prendre des mesures pour assurer l'efficacité de sa décision en évitant de causer des dommages aux coffres publics, le plus souvent en bloquant les administrations prétendument détournées ou détournées. Dans ce cas, encore une fois, sans aucune certitude, mais avec preuve d'inconduite administrative ou de mauvaise gestion des fonds publics, la Cour de Comptes peut éviter des dommages par une mesure de précaution visant à bloquer des valeurs ou des avoirs (WEDY, 2009, p. 56).

Dans le cadre de la Cours de Comptes de l'État de Rio Grande do Sul/ RS, la question est réglée dans la résolution $\mathrm{n}^{\circ} .544 / 2000$, qui établit le règlement intérieur de l'organe. ${ }^{6}$

En ce sens, il incombe au conseiller rapporteur, compte tenu de l'urgence, de prendre des mesures pour protéger le Trésor. Cependant, il est clair que, indépendamment de l'inscription à l'ordre du jour, le Conseil sera soumis à des fins de référendum.

En résumé, il est possible de démontrer fermement la possibilité d'utiliser le pouvoir général de prudence des Cours de Comptes en tant qu'instrument efficace de prévention, en évitant de nuire au Trésor et à l'environnement.

\footnotetext{
$6 \quad$ "Article 48 - Le directeur-rapporteur est chargé:

$[\ldots]$

XIII - déterminer, de toute urgence, les injonctions préliminaires du Trésor, en les soumettant à un référendum ultérieur par le Bureau compétent, indépendamment de leur inscription à l'ordre du jour;"
} 


\section{Responsabilité spéciale: garantie d'une protection efficace de l'environnement}

La gestion de procédures rapides pour protéger le trésor contre son utilisation inappropriée ou la dégradation de l'environnement doit être un impératif d'une bonne gestion.

La responsabilité spéciale est "un processus administratif exceptionnel visant à déterminer la responsabilité en cas d'omission ou d'irrégularité dans la responsabilité ou les dommages causés au trésor» (FERNANDEZ, 2009, p. 29).

Pour que la responsabilité spéciale soit possible, les ressources doivent être publiques. En outre, outre la nature de la ressource, il doit exister une destination par le biais d'un ajustement, d'une durée, d'un accord, enfin, par le biais d'un instrument qui gère l'obligation d'appliquer le montant lié à la destination spécifique de la destination.

La gestion de la responsabilité spéciale fait l'objet d'une certaine prudence. Dans la phase externe, qui doit être poursuivie devant la Cour de Comptes, après constatation du fait, dans la phase interne (devant le tribunal), le respect de la légalité doit être respecté avec l'intégralité de la défense contradictoire et large.

L'obligation de rendre des comptes découle directement de l'article 70 , seul alinéa de la Constitution Fédérale. Cela présuppose une obligation préconstituée. Les biens publics et les valeurs ne sont pas disponibles. Ils appartiennent à la collectivité et leurs administrateurs le font au nom de cette collectivité.

La responsabilité spéciale s'adresse à ceux qui ont une responsabilité découlant de la fonction de gestion, d'administration des ressources et des biens publics. La manière dont le terme donnant lieu à cette obligation sera nommé est indifférente, qu'il s'agisse d'accord, d'ajustement, de subvention, etc.

Ce qu'il faut évaluer aux fins de la possibilité de créer une responsabilité spéciale n'est pas la qualité de la personne à qui l'action doit être adressée, mais la nature de l'appel transmis. Pour cette raison, la responsabilité spéciale peut même atteindre le particulier. Le recours étant d'origine publique, toute personne, qu'elle soit juridique, publique ou privée, sera contactée par la Cour de Comptes. L'attribution de fonds ou de biens publics entraîne une application dans l'intérêt de la communauté. Toute autre destination implique une indemnisation du trésor qui peut être déterminée par le biais de la responsabilité spéciale, car la destination est l'obligation de rendre compte. C'est la seule définition que nous puissions trouver dans une analyse systématique du normatif impliquant la matière.

L'hypothèse de l'emploi de la responsabilité spéciale est un préjudice pour le trésor. II ne s'agit pas d'une vérification ordinaire des comptes. Pour cela, il y a la responsabilité normale par le processus ordinaire. Un autre aspect important à noter est que l'objectif de la responsabilité spéciale est de déterminer la perte et son indemnisation au trésor. La responsabilité spéciale ne vise pas la responsabilité dans les domaines administratif ou pénal. Pour cela, il existe d'autres instituts juridiques applicables, dans le champ administratif, le processus administratif disciplinaire, par exemple.

\section{Terme d'adoption des dispositions}

L'un des instruments à utiliser pour la mise en œuvre d'une injonction de la Cour de Comptes est le terme d'adoption des dispositions ${ }^{7}$ ou le terme d'ajustement du comportement (TAC - termo de ajustamento de conduta). C'est une compétence basée sur les points IX et $X$ de l'article 71 de la Constitution fédérale.

L'objet de ce terme est ainsi défini: "Il est possible d'adopter un instrument de la Cour de Comptes, destiné à convenir de la compétence pour adopter des mesures susceptibles d'exclure, d'atténuer ou de prévenir les irrégularités."8

Désignation utilisée par la Cour de comptes du Rio Grande do Sul dans l'avis d'audit n 14/2009: "Quant au nom de cet "accord", qui se traduit matériellement par un "terme", il convient d'utiliser la terminologie déjà existante dans le Constitution fédérale (article 71, IX): "adoption de mesures". Cela aboutirait à I 'engagement à adopter des dispositions", instrumentalisé par une "durée d'engagement à adopter des dispositions", ou, simplement, à la "durée de l'adoption des dispositions - TAP" (si l'on considère la pratique résultent d'autres mentions légales, qui traitent du "terme d'ajustement du comportement - TAC")."

8 Avis $n^{\circ}$ 14/2009. Engagement à adopter des mesures (Parecer n 14/2009. Compromisso para a adoção de providências) Disponible en: http:// www1.tce.rs.gov.br/aplicprod/f?p=50202:7:1604861591112832::NO. Accès en: 12 sept. 2011. 
Par conséquent, en termes de protection de l'environnement, en vérifiant la violation de la législation par les entités juridictionnelles et en tenant compte du domaine de compétence attribué aux cours de comptes, il est possible d'utiliser cet instrument comme un moyen d'atteindre, dans la plupart des cas dès que possible, adhésion de la direction aux normes et pratiques de protection de l'environnement, atténuation ou prévention de la dégradation.

Dans le même ordre d'idées, nous apprenons la leçon de Vittorio Constantino Provenza, pour qui la modification de la durée de la conduite constitue une nouvelle alternative à la disposition du contrôle externe dans l'exercice de ses fonctions constitutionnelles (PROVENZA, 2007, p. 64-70). Et il parvient à cette conclusion en utilisant la théorie des pouvoirs implicites, car si une certaine fonction lui est attribuée, il faut considérer que tous les instruments nécessaires et appropriés ont été accordés pour la réalisation de cette mission. Et rien de plus efficace que la possibilité que les dommages cessent dès qu'ils sont détectés lorsqu'il s'agit de problèmes environnementaux.

Le terme d'ajustement du comportement (TAC - termo de ajustamento de conduta) est l'instrument qui vise naturellement à reconnaître l'illégalité d'un acte donné et la présomption concomitante, devant l'organisme public légitime de surveillance du comportement, d'un engagement d'adapter le comportement du contrevenant aux exigences juridiques afin de: éliminer la situation d'illégalité (MILARÉ, 2009, p. 488).

La loi sur les enchères contient une disposition similaire qui permet une protection préventive. Justen Filho souligne que la Cour Suprême a reconnu, dans l'arrêt MS 24.510, que la Cour de Comptes de Fédérale (TCU) avait des compétences de nature préventive (JUSTEN FILHO, 2008, p. 867).

Par conséquent, on peut voir que le terme d'adoption des dispositions est un instrument qui peut être utilisé dans la protection de l'environnement, corrigeant l'orientation des politiques dans ce domaine grâce à l'engagement pris par les administrateurs et soumis à une application forcée.

\section{Négatif de la force exécutoire d'une loi ou d'un acte normatif}

Sur la base du précédent $n^{\circ} 347$ de la Cour Suprême Fédérale, ${ }^{9}$ les Cours de Comptes peuvent (comme il se doit) examiner la constitutionnalité des lois et des actes du pouvoir public. En ce sens, en utilisant le responsable de l'acte normatif pour fonder l'agression sur l'environnement, contre les dispositions de la Constitution, la défense du bien environnemental peut être garantie par la déclaration d'inconstitutionnalité de la loi ou l'acte normatif à l'examen.

Le sujet est régi par le Règlement intérieur de la Cour de Comptes Fédérale (TCU), qui prévoit que la Plénière décide d'un conflit de loi ou d'un acte normatif du pouvoir public avec la Constitution fédérale en ce qui concerne la compétence de la Cour. ${ }^{10}$

Il est important de comprendre la nature et les effets de cette décision. En ce sens, la Cour de Comptes doit se prononcer sur la base de la déclaration du précédent de la Cour Suprême 347, de la même manière

\footnotetext{
La Cour de comptes, dans l'exercice de ses compétences, peut apprécier la constitutionnalité des lois et actes de pouvoir public

"Règlement intérieur de la Cour fédérale de comptes. Résolution 155, du 4 décembre 2002.

Art. 15. La Plénière, dirigée par le Président de la Cour, est exclusivement chargée:

Je - délibère à l'origine sur:

$[\ldots]$

e) conflit de loi ou acte normatif de la puissance publique avec la Constitution fédérale, concernant la compétence de la Cour;

D'autres tribunaux ont également inclus des dispositions similaires dans leurs règlements. Il est mentionné, par tous:

Règlement intérieur de la Cour des comptes de l'État de Rio Grande do Norte.

Incidents d'inconstitutionnalité

Article 274. La Cour des comptes, dans l'exercice de ses attributions, peut statuer sur l'inconstitutionnalité des lois et actes de la puissance publique. Article 275. Si, au moment du jugement de quoi que ce soit rendu par les Chambres, elles concluent qu'une loi ou un acte de la Puissance publique est inconstitutionnel, le dossier sera transmis sans jugement sur le fond à la Cour plénière afin qu'elle puisse statuer à titre préliminaire sur la question. [...] § 3. Jugée par la Cour et la décision correspondante publiée, l'affaire sera renvoyée à la chambre pour qu'elle l'apprécie conformément à la décision préjudicielle d'inconstitutionnalité.

§ 4. La décision rendue par les deux tiers des membres de la Cour qui concluent en refusant le respect de la loi ou de l'acte jugé inconstitutionnel constituera, pour l'avenir, une règle définitive et d'application obligatoire, dans des cas similaires, à moins que la Chambre, le conseiller ou le procureur du ministère public spécial pour des raisons pertinentes, jugent nécessaire de provoquer une nouvelle décision de la Cour plénière sur la question."
} 
que celle publiée par le Sénat fédéral sur la base de l'art. 52. X, atteint le plan d'efficacité de la décision. L'appareil lui-même porte l'expression "suspendre l'exécution" afin de caractériser cette compréhension.

Cela a un effet extrêmement important, car si la loi est jugée constitutionnelle par la Cour suprême, elle produira à nouveau ses effets. Si l'entente était différente, il n'y aurait pas de telle possibilité.

Par conséquent, le déni de force exécutoire fondé sur le Précédent $n^{\circ} 347$ de la Cour Suprême Fédérale a pour effet de suspendre l'effectivité de l'acte administratif et des actes normatifs qui le soustendent (SCHMITT, 2006, p. 172). Les normes restent dans le système, mais sans effet jusqu'à l'intervention du pouvoir judiciaire, qui en décidera.

Le déni de force exécutoire est une obligation convaincante pour les cours de comptes. Ici, nous sommes confrontés à un devoir, ou pouvoir-devoir, imposé à ces organes en tant que gardiens des droits fondamentaux et garanties dans le traitement des affaires publiques. En ce sens, susciter la permanence des effets d'un droit anticonstitutionnel n'est pas remplir sa mission institutionnelle et revêtir une importance aussi significative pour le maintien de l'état de droit (SCHMITT, 2006, p. 175).

Par conséquent, s'il existe une loi anticonstitutionnelle contraire aux ordres énoncés à l'article $225 \mathrm{du}$ Constitution Fédérale, cette législation aurait pu / aurait dû nier son efficacité, son caractère exécutoire en tant que moyen de préserver la norme constitutionnelle.

Un autre point à noter est la clause de réserve plénière (full bench), fondée sur l'article 97 de la Constitution Fédérale. ${ }^{11}$ La question était soumise à l'examen de la Cour Suprême Fédérale, qui avait publié le précédent contraignant $\mathrm{n}^{\circ} 10 .{ }^{12}$ Les cours de comptes sont donc soumises à sa conformité, car l'exigence de la clause de réserve plénière (full bench) est également déterminante lorsque la décision d'un organe juridictionnel fractionné ne fait que supprimer son incidence, en totalité ou en partie.

Aux termes de la Constitution Fédérale de 1988, depuis son origine, il est toujours nécessaire de déclarer inconstitutionnelle une loi ou un acte normatif, que cette décision soit prise à la majorité absolue des membres des tribunaux. Les organes spéciaux des tribunaux, où ils sont constitués, bénéficient également de cette possibilité.

L'objet de la réserve plénière est la présomption de constitutionnalité des lois et des actes normatifs, selon laquelle la règle est sa constitutionnalité, l'inconstitutionnalité étant l'exception, l'anomalie, qui ne devrait être déclarée/vérifiée que dans les cas où elle ne l'est pas. II n'y a aucune possibilité de préserver la norme juridique préparée par le législateur. Par conséquent, l'objectif de la règle du full bench, en exigeant le quorum requis des membres des organes collégiaux pour des décisions de cette nature, signifie "la prudence de l'électeur lorsqu'il traite d'une question d'une si grande ampleur que la déclaration d'inconstitutionnalité". (BITTENCOURT apud AMARAL JÚNIOR, 2002, p. 44).

En ce sens, l'application de la clause de réserve en plénière par la Cour de Comptes est obligatoire. La décision rendue a pour effet d'exclure l'incidence, en tout ou en partie, d'une loi ou d'un acte normatif agissant sur l'efficacité de la règle, et non son retrait de l'ordre juridique, comme c'est le cas de la déclaration d'inconstitutionnalité par le Pouvoir Judiciaire (nullité).

Lors de l'analyse de la déclaration du Précédent $n^{\circ} 347$, Roberto Rosas a déclaré qu'il incombait à la Cour de comptes: de l'interprétation privée des lois par le pouvoir judiciaire (ROSAS, 1998, p. 137).

Le résultat de l'examen de la Cour, dû à l'inconstitutionnalité de la loi ou de l'acte normatif, conduit à son refus d'application, car "il faut distinguer entre déclarer l'inconstitutionnalité et ne pas appliquer de lois inconstitutionnelles, puisqu'il s'agit de l'obligation de tout tribunal ou organe de quelque nature que ce soit des pouvoirs de l'État" (ROSAS, 1998, p. 138).

11 "Art. 97. Ce n'est que par le vote de la majorité absolue de ses membres ou des membres de l'organe spécial concerné que les tribunaux peuvent déclarer l'inconstitutionnalité d'une loi ou d'un acte normatif du pouvoir publique."

12 Résumé contraignant $n^{\circ} 10$, publié le 27 juin 2008, dont la diction est la suivante: Il viole la clause de réserve plénière (CF, art. 97 ) la décision d'un organe judiciaire fractionnaire qui, bien qu'il ne déclare pas expressément l'inconstitutionnalité d'une loi ou d'un acte normatif de la puissance publique, en supprime en tout ou en partie l'incidence. 
Par conséquent, le refus du caractère exécutoire d'une loi ou d'un acte normatif pouvant entraîner la suspension de son efficacité à titre de mesure de précaution est une procédure d'efficacité d'une injonction environnementale reconnue par la Cour Suprême Fédérale et extrêmement efficace pour la protection de l'environnement.

Avec les autres instruments mentionnés, la négation de la force exécutoire d'une loi ou d'un acte normatif est un moyen efficace de prévention et de protection du bien environnemental, empêchant les dommages déjà causés de se multiplier et de devenir de plus en plus dommageables.

\section{La performance de la Cour de Comptes vers la protection de l'environnement}

La Cour de Comptes Fédérale, au processus rapporté par le Ministre Aroldo Cedraz, a jugé le suivi des actions gouvernementales visant à enquêter sur la mise en œuvre des mesures proposées par la Cour des Comptes en faveur de l'atténuation et de l'adaptation des secteurs de l'économie aux changements climatiques.

Le suivi de ces questions est lié aux propositions de l'Union en matière environnementale. De cette manière, il a été discuté du processus de mise en œuvre de mesures telles que la préservation de la forêt amazonienne, la sécurité des ressources en eau, l'attribution d'un soutien économique au secteur agricole, entre autres.

Le rapport d'audit opérationnel (affaire $n^{\circ} 026.099 / 2008-3^{13}$ ) visait à évaluer le rôle du gouvernement fédéral vers la réduction des gaz à effet de serre dans la région amazonienne. Au cours du processus, des lacunes ont été notées et des recommandations ont été faites.

Dans le jugement 2293, ${ }^{14}$ par l'arrêt du 30/09/2009, il a abouti à une suggestion d'élaborer un calendrier d'actions dans le PNACC - Plan National d'Adaptation au Changement Climatique, à réaliser par le Comité interministériel et le Groupe exécutif sur le changement climatique. Pourtant, à cette occasion, il a été recommandé d'utiliser des outils économiques pour promouvoir les producteurs ruraux en contact avec la préservation et la récupération des forêts, ainsi que la diffusion technique et la formation nécessaires. Ces outils visaient l'ouverture de lignes de crédit par le Ministère de l'Agriculture, de l'Élevage et de l'Approvisionnement - Ministério da Agricultura, Pecuária e Abastecimento (MAPA).

En outre, il a été recommandé à l'Institut National de Colonisation et de Réforme Agraire (INCRA) d'adopter les mesures établies par le Plan d'action pour l'environnement, d'adapter les conditions de la Département du développement de l'Amazonie (SUDAM) par le Ministère de l'Intégration Nationale. II a été suggéré au SUDAM de réévaluer la liste des priorités et des lignes directrices pour l'allocation des ressources du Fonds de développement de l'Amazonie, qui devrait être conforme aux dispositions du Plan d'Amazonie de Développement Durable, conduisant ainsi à la définition des ressources budgétaires en la matière. En outre, il a affirmé au Ministère de l'Environnement qu'il rendrait opérationnelle, par le biais du Portail National de Gestion Forestière, la distinction entre la déforestation légale des illégales.

II est clair que la liste des recommandations formulées dans l'arrêt n'a pas été épuisée. Cependant, l'Action de suivi 021.295/2018-2 a révélé que certaines mesures proposées étaient en cours de mise en œuvre, telles que des modifications des lignes de crédit au secteur de la production rurale pour la plantation de forêts plantées et la récupération des réserves légales et des APP (zone de conservation permanente). Le programme en question s'appelait le Plan ABC (Plan pour une agriculture à faibles émissions de carbone), reconnaissant le soutien technique aux producteurs et favorisant une agriculture durable, comprenant ainsi le respect de la recommandation 9.2.1 du jugement 2.293/2009. En ce qui concerne l'adéquation et la

13 “Cour fédérale de comptes - TCU. Tribunal de Contas da União. Controle e Acompanhamento de Processos do TCU. Relatório de Auditoria: Processo: 026.099/2008-3. TCU, 22 set. 2008. Disponible en: https://contas.tcu.gov.br/etcu/AcompanharProcesso?p1=26099\&p2=2008\&p3=3. Acèss en: 21 septembre. 2020.

14 “Cour fédérale de comptes - TCU. Tribunal de Contas da União. Acórdão: 2293/2009. Relatório de Auditoria: 026.099/2008-3. [S. I.], 30 set. 2009. Disponible en: https://pesquisa.apps.tcu.gov.br/\#/documento/acordao-completo/*/NUMACORDAO\%253A2293\%2520ANOACORDAO\%2 53A2009/DTRELEVANCIA\%2520desc\%252C\%2520NUMACORDAOINT\%2520desc/0/\%2520?uuid=21b47180-fdd0-11ea-8a93-ef11eab550bd. Acèss en: 21 septembre. 2020. 
définition des ressources destinées au Fonds de développement de l'Amazonie, l'action a considéré que la recommandation n'était pas mise en œuvre.

L'action de suivi visait à analyser le non-respect total, partiel ou non des autres arrêts. Toutes les recommandations ont été formulées dans des audits dont l'objectif était d'intervenir dans l'administration publique en faveur des adaptations du secteur économique dans l'atténuation des effets du changement climatique.

Le jugement 2.354/2009, résultat du processus 026.158/2008-6, a également été discuté dans l'action de suivi. Le processus a également porté sur un rapport d'enquête d'audit opérationnel, cette fois en revue des actions d'atténuation du changement climatique dont les conséquences menacent l'élévation du niveau de la mer. Des carences dans les politiques publiques nationales ont également été relevées et, dans ce sens, des recommandations ont été faites à l'Agence nationale des transports fluviaux (ANTAQ), au Ministère de l'Environnement, au Conama (Conseil national de l'environnement), au Conseil ressources nationales en eau et huitième Secrétariat du contrôle externe.

Les recommandations des ministres de la Cour de Comptes Fédérale concernaient plusieurs aspects déficitaires des politiques publiques. Parmi eux, nous soulignons la mise en place d'un système de suivi permanent des variables océaniques, identifiant les zones vulnérables et les zones côtières à risque, en attribuant la responsabilité aux instances compétentes. L'action de suivi a examiné la mesure de mise en œuvre.

La recommandation considérait également que l'activité portuaire serait discutée dans l'agenda du changement climatique par l'ANTAQ et le Ministère de l'Environnement, ce qui a été considéré comme mis en œuvre par les audits réalisés. Pourtant, en termes de recommandations, Conama a été chargé d'élaborer des lignes directrices pour l'octroi de licences environnementales des zones côtières, étant donné le degré élevé d'impact résultant du changement climatique. La recommandation n'apparaît pas comme une pratique de mise en œuvre et n'est pas non plus mise en œuvre selon le jugement 73/2020.

Des politiques gouvernementales visant au secteur agricole ont été suggérées par le jugement 2.513/2009, adressé à l'Agence liée directement au président de la république (Casa Civil), pour promouvoir des actions de sensibilisation des gestionnaires publics aux politiques publiques visant le secteur agricole, en effets du changement climatique, il a également recommandé la disponibilité de données météorologiques à court et à long terme, entre autres recommandations aux agences axées sur la météorologie, pour l'exactitude et l'évaluation des données météorologiques. Les deux mesures ont été considérées comme mises en œuvre pendant le processus de surveillance.

Ainsi, ce sont quelques exemples de la performance de la Cour de Comptes Fédérale, en ce qui concerne les mesures de protection de l'environnement.

\section{Conclusion}

L'analyse des questions environnementales relève de la compétence des cours de comptes et nécessite une action efficace pour prévenir les dommages en recherchant le maximum de protection. Une protection inhibitrice de la protection de l'environnement suppose l'action rapide des organes de l'État chargés de la surveillance et du contrôle des entités fédérées dans la formulation et l'exécution de leurs tâches constitutionnelles. Par conséquent, il est nécessaire que les comités techniques établissent dans les normes qui organisent leurs activités des mécanismes qui permettent de le réaliser de cette manière.

Outre la création et l'amélioration des instruments à sa disposition, les cours de comptes doivent avoir recours à des mécanismes de contrôle, tels que la responsabilité spéciale, l'application du pouvoir général de prudence, l'appel à la consommation durable, la demande de dossier d'appel d'offres, la régularité des procédures environnementales, l'application du terme de providence, ainsi que les déterminations. Tous ces instruments sont déjà à la disposition de ces organismes, qui ont le devoir de promouvoir la culture d'une protection inhibitrice afin de garantir la protection de l'environnement et, par conséquent, la qualité de la vie.

II convient de noter qu'il y a une grande évolution en ce qui concerne les questions intéressant les cours de comptes, exigeant des professionnels travaillant dans ce domaine une compréhension plus approfondie 
du champ d'action, de la compétence et des limites de ces organes. Le besoin découle en particulier d'une transformation de la société qui exige de plus en plus d'efficacité et de résultats des organismes publics.

La Cour de Comptes Fédérale étudie actuellement un "Code de procédure des Cours de Comptes" ayant une incidence nationale. Nous pensons que certaines des questions soulevées ici pourraient intégrer ce texte, ainsi que le développement d'autres, afin d'uniformiser davantage les procédures à adopter, dans le but de protéger efficacement l'environnement et d'appliquer correctement les ressources publiques dans ce domaine.

\section{Bibliographie}

AMARAL JÚNIOR, José Levi. Incidente de Argüição de Inconstitucionalidade - Comentários ao art. 97 da Constituição e aos arts. 480 a 482 do Código de Processo Civil. São Paulo: Revista dos Tribunais, 2002.

FERNANDEZ, Jorge Ulisses Jacoby. Tomada de contas especial. 4. ed. Belo Horizonte: Fórum, 2009. JUSTEN FILHO, Marçal. Comentários à Lei de Licitações e Contratos Administrativos. 12. ed. São Paulo: Dialética, 2008.

MARINONI, Luiz Guilherme; MITIDIERO, Daniel. Código de processo civil. São Paulo: Revista dos Tribunais, 2008.

MILARÉ, Édis. Direito do ambiente. 2. ed. São Paulo: Revista dos Tribunais, 2009.

PROVENZA, Vittorio Constantino. Controle externo. Uma nova alternativa: possibilidade de utilização do termo de ajustamento de conduta pelos Tribunais de Contas no exercício de suas funções constitucionais. Revista do TCMRJ, Rio de Janeiro, v. 24, n. 35, p. 64-70, maio 2007.

RIZZO JUNIOR, Ovídio. Controle social efetivo das políticas públicas. 2009. Tese (Doutorado em Direito Econômico e Financeiro) - Faculdade de Direito, Universidade de São Paulo, São Paulo, 2009. Disponível em: https://bdtd.ibict.br/vufind/Record/USP_666fea1bdc02caf6313c70a748e42c04. Acesso em: 08 jun. 2011.

ROSAS, Roberto. Direito sumular: comentários às súmulas do Supremo Tribunal Federal e do Superior Tribunal de Justiça. 9. ed. São Paulo: Malheiros, 1998.

SARLET, Ingo Wolfgang; FENSTERSEIFER, Tiago. Estado socioambiental e mínimo existencial (ecológico?): algumas aproximações. In: SARLET, Ingo Wolfgang (org.). Estado socioambiental e direitos fundamentais. Porto Alegre: Livraria do Advogado, 2010. p. 11-38.

SCHMITT, Rosane Heineck. Tribunais de contas no Brasil e controle de constitucionalidade. 2006. Tese (Doutorado em Direito) - Programa de Pós-graduação em Direito, Universidade Federal do Rio Grande do Sul, Porto Alegre, 2006. Disponível em : https://bdtd.ibict.br/vufind/Record/URGS_1921b4e9a e96ebbb0e1c93ea7c26999f. Acesso em: 03 jun. 2011.

STONOGA, Andreza Cristina. Tutela inibitória ambiental: a prevenção do ilícito. Curitiba: Juruá, 2007.

WEDY, Gabriel. O princípio constitucional da precaução como instrumento de tutela do meio ambiente e da saúde pública. Belo Horizonte: Fórum, 2009.

Recebido em: 01/01/2020

Aprovado em: 14/09/2020 